\title{
Tauchbeobachtungen an Plankton und an Echostreuschichten
}

\author{
Jürgen Lenz und Hjalmar Thiel \\ Institut für Meereskunde der Universität Kiel, Kiel, \\ und \\ Institut für Hydrobiologie und Fischereiwissenschaft \\ der Universität Hamburg, Hamburg
}

\begin{abstract}
Diving observations on plankton and on scattering layers. Diving techniques are employed as a research tool in plankton investigations carried out in shallow water of the western Baltic Sea. Observations and samplings were made by skin divers on scattering layers corresponding to the discontinuity layers. Biogene materials, sometimes concentrated at the thermocline, are not responsible for this special kind of scattering, but rather discontinuity of salinity and temperature (LeNz 1965). For observations in deep water the use of undersea vehicles is recommended. From the Bathyscaph and the diving saucer, single plankton organisms and plankton concentrations were observed (e. g. Bernard 1958); investigations on the deep scattering layer have shown physonectid siphonophores and myctophids to be scatterers (BARHAM 1966). The equipment for sampling plankton and benthos from undersea vehicles is poorly developed. We need urgently gear for quantitative and qualitative sampling and for manifold use during single dives, i. e., multiple sampling gear and magazins for storage of samples.
\end{abstract}

\section{EINLEITUNG}

Bei der Durchsicht der Literatur ist festzustellen, daß sich nahezu alle hydrobiologischen Arbeiten, bei denen die Methode des Tauchens eingesetzt wurde, mit Problemen des Benthos befassen. Nur einzelne Veröffentlichungen beziehen sich auf Beobachtungen an Plankton und an Echostreuschichten. Daher sollen Methoden und Ergebnisse solcher Untersuchungen beschrieben werden, bei denen Schwimmtaucher und Tauchboote eingesetzt worden sind. Abschließend sollen einige Forderungen für die Entwicklung von Geräten vorgetragen werden, durch die Tauchboote in der biologischen Forschung erst voll befriedigend Verwendung finden können.

\section{BEOBACHTUNGEN BEIM SCHWIMMTAUCHEN}

Im Jahre 1961 bildete sich an der Universität Kiel eine Tauchgruppe, die sich mit verschiedenen meereskundlichen Problemen befaßte. Während unserer Arbeiten unter 
Wasser machten wir die ersten Beobachtungen über die Schichtung von Wassermassen: (1) Die durch Plankton und Detritus getrübte Oberschicht setzte sich scharf von der klaren Unterschicht ab. (2) Zwischen der nicht getrübten Ober- und Unterschicht verlief eine etwa handbreite, stark getrübte Schicht, die nach oben und unten scharf abgegrenzt war. (3) Beim Abtauchen konnten mit den ungeschützten Händen Temperaturunterschiede bis $\mathrm{zu} 2^{0} \mathrm{C}$ gefühlt werden. (4) Unter bestimmtem Winkel lassen sich wegen der Totalreflektion solche Grenzschichten nicht durchschauen. (5) Beim

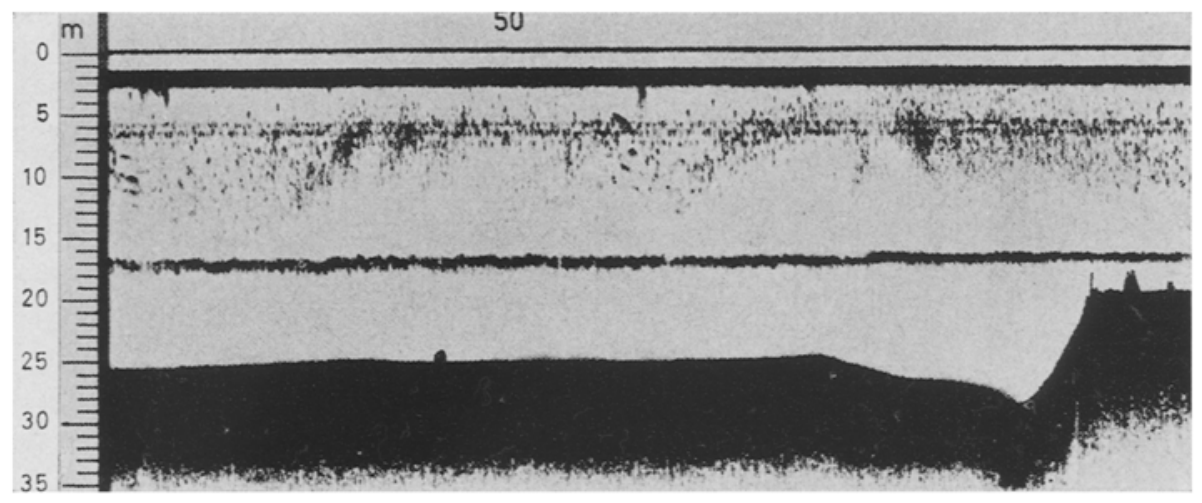

Abb. 1: Echogramm mit einer starken Streuschicht in $17 \mathrm{~m}$ Tiefe, wie man es in der westlichen Ostsee regelmäßig aufnehmen kann. (Original von LENZ)

Vermischen der Schichten bilden sich Schlieren. (6) Eindeutig zu identifizierende Individuen aus dem Großplankton fanden sich nur in einer der beiden Schichten: so beispielsweise die Hydromeduse Halitholus cirratus und die Ctenophore Bolinopsis infundibulum.

Für Beobachtungen dieser Art eignet sich die westliche Ostsee besonders gut. Die aus dem Skagerrak und dem Kattegat durch den Großen Belt in die westliche Ostsee eindringenden Wassermassen füllen die tiefen Rinnen und Becken und werden gleichzeitig von dem salzärmeren Wasser aus der östlichen Ostsee überschichtet. Zu bestimmten Jahreszeiten wird diese Schichtung durch einen gleichsinnigen Temperatureffekt verstärkt, so daß sich eine recht stabile Schichtung herausbildet, die vom Echographen deutlich aufgezeichnet wird (Abb. 1).

Zur Zeit unserer ersten Beobachtungen von Wasserschichten hatte LeNZ (1965) seine Untersuchungen über die Ursache der an die Sprungschicht gebundenen Echostreuschichten in der westlichen Ostsee begonnen, und es stellte sich die Frage, ob es nicht sinnvoll wäre, parallel $\mathrm{zu}$ den herkömmlichen Methoden mit Bathythermograph, Durchsichtigkeitsmeßgerät, Wasserschöpfer, Planktonpumpe, Netz und Planktonröhre die Schichtung selbst zu beobachten und tauchend gezielte Proben aus den verschiedenen Schichten zu entnehmen.

Dabei standen wir zunächst vor dem Problem, die gewünschte Schicht antauchen zu können, ohne sie jedoch vor der Probennahme zu zerstören. Es hatte sich nämlich gezeigt, daß die Trübungsschirme nur zu erkenmen waren, wenn man sich unter ihnen befand und schräg gegen sie, die Wasseroberfläche und den hellen Himmel blickte. Die 
Probennahme hatte also von unterhalb der zu untersuchenden Schicht zu erfolgen. Auch wenn die Schichtungen bemerkenswert stabil waren und sie nur in dem Bereich verwirbelt wurden, in dem der Taucher sie durchstieß, so war die Probennahme doch durch die aufsteigenden Blasen der ausgeatmeten Luft behindert. Es war daher not-

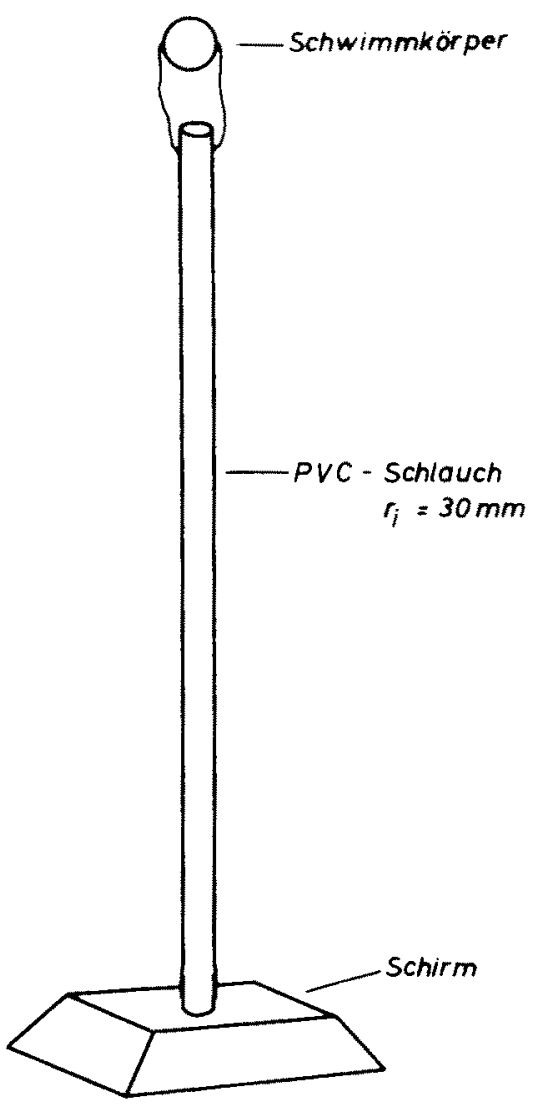

Abb. 2

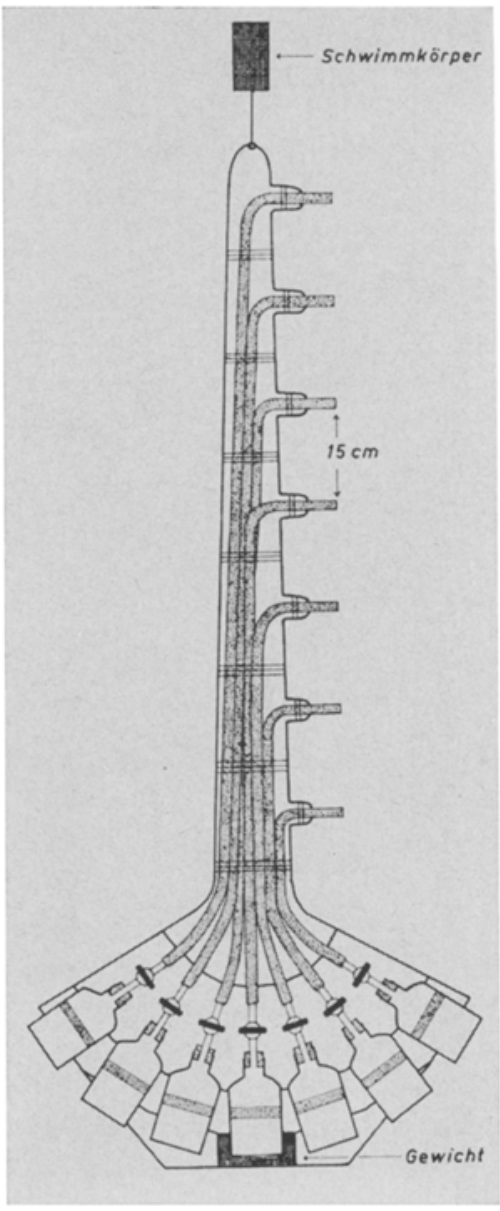

Abb. 3

Abb. 2: Zusatzapparatur zum Tauchgerät zur Ableitung der ausgeatmeten Luft. Der Schlauch ist $2 \mathrm{~m}$ lang und hat einen Innendurchmesser von $60 \mathrm{~mm}$. (Original)

Abb. 3: Gerät zur Entnahme von Proben aus bestimmten Wasserschichten. Die Probennahme geschieht damit nahezu gleichzeitig und in gleichmäßigen Abständen. (Nach LENZ 1965)

wendig, die ausgeatmete Luft so zu leiten, daß sie erst oberhalb der Schichtung ins freie Wasser gelangte. Als Zusatzgerät nahmen wir einen weiten Schirm, der über der Austrittsöffnung der ausgeatmeten Luft befestigt wurde und die Blasen auffing. An diesen war über einen Stutzen ein $2 \mathrm{~m}$ langer Schlauch aus weichem Polyvenylchlorid mit einem Innendurchmesser von $6 \mathrm{~cm}$ angeschlossen. Um den Schlauch unter Wasser senk- 
recht zu halten, wurde an seinem oberen Ende eine Fischerkugel angebunden (Abb. 2). Der Taucher konnte sich nun dicht unter der Schicht aufhalten, der Schlauch durchstieß diese zwar, zerstörte sie aber nicht. Die Blasen der verbrauchten Atemluft traten so erst etwa $1,5 \mathrm{~m}$ über der Schicht aus. Auch beim Schwimmen und Abtauchen behinderte diese Anordnung den Taucher nicht, da die Luftblasen bei horizontaler Schwimmlage unter dem Schirmrand heraustreten konnten.

Das zweite technische Problem, das gelöst werden mußte, war die Probenentnahme unter Wasser und aus eng begrenzten Schichten. Wir arbeiteten zunächst mit einzelnen großen, evakuierten Flaschen, deren Ansaugstutzen in die gewünschten Schichten hin-

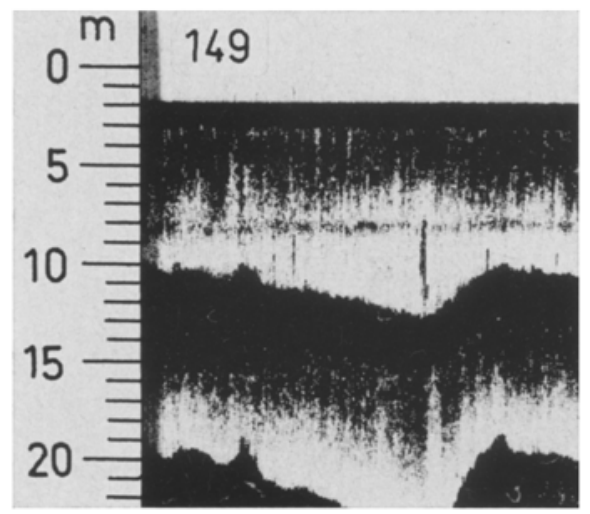

Abb. 4: Echogramm mit einer schwachen Streuschicht in $8 \mathrm{~m}$ Tiefe. (Original von LENZ)

eingehalten wurden. $\mathrm{Da}$ der Taucher aber jeweils nur eine dieser Flaschen füllen konnte, entwickelte LENZ (1965) ein einfaches Gerät. Es besteht aus 7 Flaschen, die ebenfalls evakuiert wurden und deren Ansaugstutzen in regelmäßigen Abständen übereinander - in diesem Falle $15 \mathrm{~cm}$ - angeordnet waren (Abb. 3). Der Taucher hält das Gerät zur Probennahme im Arm, schwimmt es von unten her in die Schicht, die untersucht werden soll, hinein und öffnet möglichst schnell nacheinander die Hähne der Flaschen. Die Probennahme geschieht auf diese Weise nahezu gleichzeitig und in definierten Abständen.

Die Ergebnisse dieser Untersuchungen sollen an einem Beispiel kurz erläutert werden: Die Entnahme von Proben direkt aus der Sprungschicht sollte vor allem Antwort auf zwei Fragen geben: (1) Wie scharf ist der von Temperatur und Salzgehalt erzeugte Dichtesprung und (2) beeinflußt die Ansammlung suspendierter Teilchen in der Sprungschicht die Aufzeichnung des Echogramms? Abbildung 4 zeigt das Echogramm einer schwachen Echostreuschicht in $8 \mathrm{~m}$ Tiefe. Sichtbare Schlieren beim Vermischen der beiden Schichten mit der Hand deuteten auf starke Dichteunterschiede hin, der Temperatursprung von $4^{0} \mathrm{C}$ konnte mit dem Bathythermographen gemessen werden und war mit der Hand deutlich spürbar, und die Zunahme des Salzgehaltes um 2\%o im Tiefenabstand von $15 \mathrm{~cm}$ wurde nachträglich an den entnommenen Proben gemessen (Abb. 5). Das Wasser über der Sprungschicht schien etwas weniger trübe zu sein als das darunter. In der Häufigkeit der suspendierten Teilchen und des Planktons in den Proben aus den 
verschiedenen Tiefenstufen ließen sich jedoch keine eindeutigen Unterschiede feststellen. Das Echo wurde in diesem Fall also allein von der scharfen Dichteschichtung erzeugt.

Die bisher vorgetragenen Beobachtungen beziehen sich auf Echostreuschichten, die unmittelbar von der Sprungschicht hervorgerufen wurden. Sie liegen im Untersuchungs-

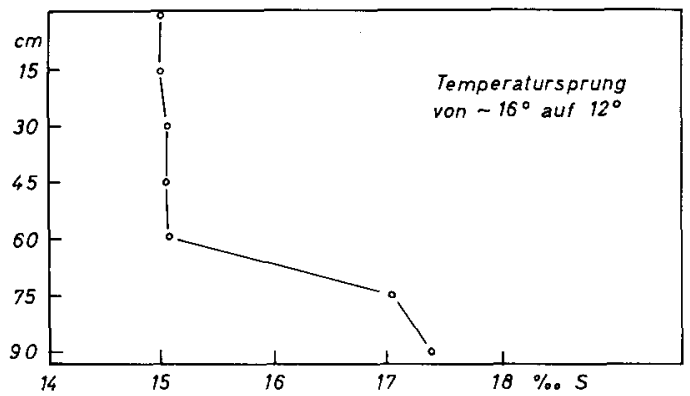

Abb. 5: Darstellung des Salzgehaltssprunges der in Abbildung 4 wiedergegebenen Streuschicht, der $2 \%$ bei einer Tiefenzunahme von $15 \mathrm{~cm}$ beträgt. (Nach LENZ 1965)

gebiet vorwiegend in Tiefen zwischen 8 und $15 \mathrm{~m}$ und können folglich vom Schwimmtaucher mit der normalen Preßlufteinrichtung leicht erreicht und untersucht werden. Vor uns haben bereits Limbaugh \& Rechnitzer (1955) und Banner (1955) Schichtungserscheinungen freitauchend beobachtet.

\section{BEOBACHTUNGEN BEIM TAUCHEN MIT TAUCHBOOTEN}

Weitere Beobachtungen an Plankton und an Echostreuschichten sind in tiefem Wasser von Tauchbooten aus vorgenommen worden. Beim Abtauchen des Bathyscaphs mit gleichmäßiger Geschwindigkeit konnten kontinuierliche Beobachtungen ausgeführt und Zählungen von Individuen vorgenommen werden. So berichten BERNARD (1958, 1962), Dietz (1959), Pérès (1958a, b), Piccard \& Dietz (1957) und Tregouboff $(1958,1962)$ von ihren Tauchgängen über einzelne Arten vom Großplankton, von Plankton- und Sestonkonzentrationen in bestimmten Tiefenhorizonten (Abb. 6), von Planktonwolken und von einer klaren Wasserschicht über dem Meeresboden.

Eine unmittelbare Untersuchung tiefer Echostreuschichten ist von diesen Autoren jedoch nicht vorgenommen worden. Diese geschieht meist mit horizontal und vertikal fangenden Schließnetzen, die mit Hilfe von fernmeldenden Tiefenschreibern in die Tiefe hineingesteuert werden, in der auf dem Echolot die Schicht angezeigt ist. In das Echogramm (Abb. 7) von einer Tiefenstreuschicht ist die Schleppstrecke einer Planktonröhre eingezeichnet worden, die mit einem fernmeldenden Tiefenschreiber ermittelt worden war (KINZER, in Vorbereitung). Von den Unsicherheiten, die in dieser Methode gegeben sind, seien folgende erwähnt: (1) Siphonophoren, die als Reflektierer erkannt worden sind (BARHAM 1963a), werden beim Fang mit den herkömmlichen Methoden leicht beschädigt und unkenntlich. (2) Die Tiere der verschiedenen Größenordnungen werden aus der befischten Schicht gefangen, doch ist damit nicht sicher festgestellt, ob 
sie alle den Schall reflektieren. (3) Die Genauigkeit von Echoaufzeichnungen und die Steuerung eines Gerätes stimmen häufig nicht überein. (4) Fanggeräte haben Scheuchwirkungen und fischen selektiv. (5) Die Aussagen über die tatsächliche Position der Tiere im Wasser sind beschränkt, weil durch ein Fanggerät immer eine Integration ihrer Konzentration über die Schleppstrecke erfolgt.

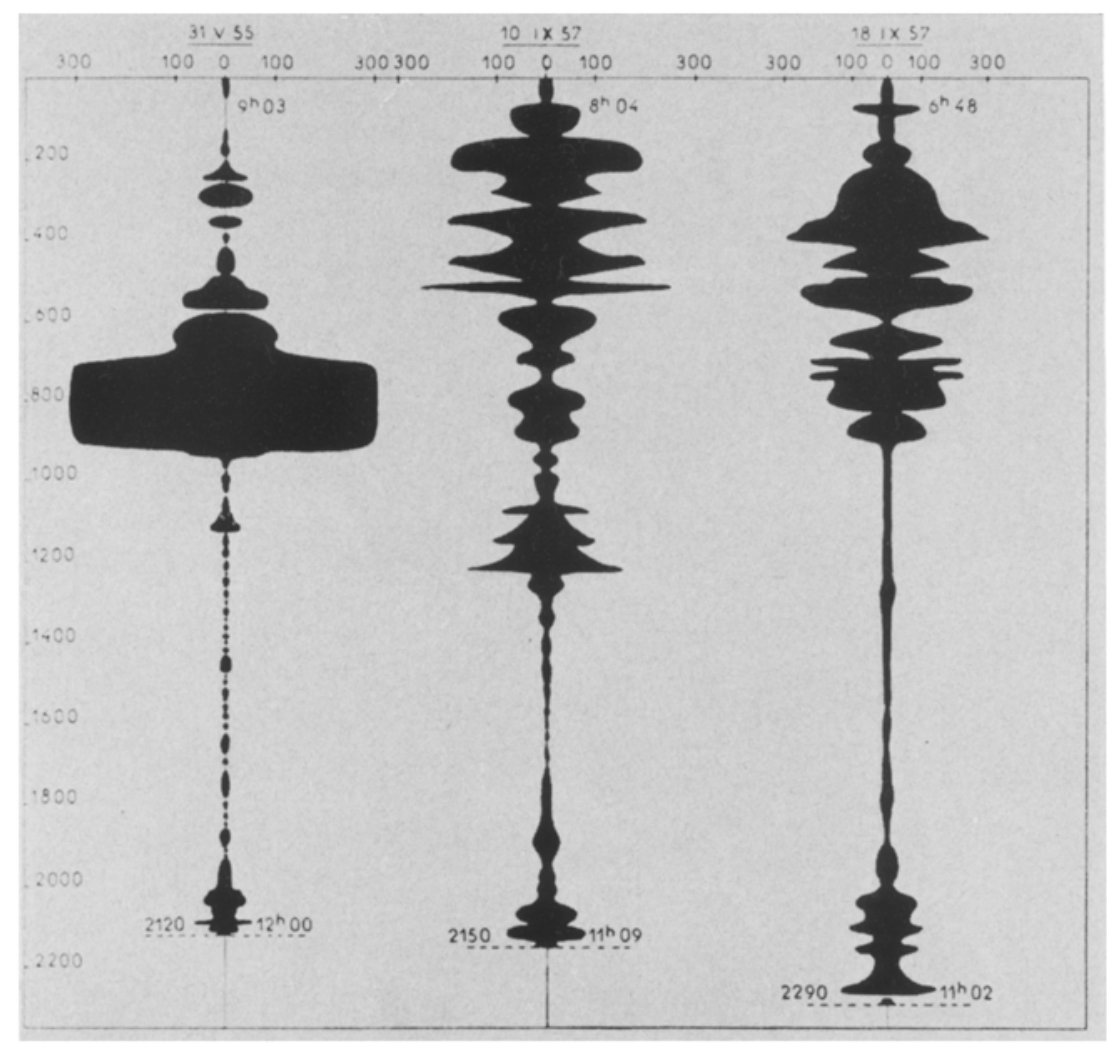

Abb. 6: Quantitative Tiefenverbreitung von Planktonorganismen nach Beobachtungen auf drei Tauchgängen mit dem Bathyscaph. (Nach BERNARD 1958)

Einige dieser Nachteile hat BARHAM (1963b, 1966a, b) dadurch umgehen können, daß er die tiefe Echostreuschicht mit Tauchbooten antauchte und die Tiere direkt beobachtete, die als Erzenger des Echos in Frage kamen. Diese Untersuchungen sind mit dem Bathyscaph „Trieste“ (1963b) und mit der Soucoupe Plongeante (1966a, b) durchgeführt worden. Aus Abbildung 8 werden Baryams Ergebnisse besonders deutlich, in der über das Echogramm der Tiefenstreuschicht, das vom Begleitschiff aufgenommen worden ist, das Protokoll der Beobachtungen gezeichnet wurde. Der Abstieg mit dem Tauchboot begann kurz nach $16^{00} \mathrm{Uhr}$, und das Boot sank zunächst mit eingeschaltetem Licht durch eine Schicht diffuser Schallreflektion bis in etwa $100 \mathrm{~m}$ Tiefe. Die Hauptbeobachtung fand in dieser Tiefe statt, so daß die zur Oberfläche aufsteigenden schall- 


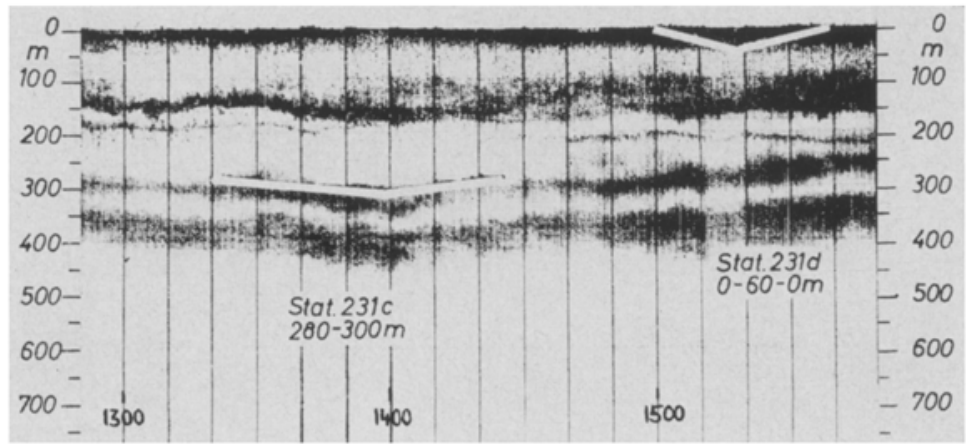

Abb. 7: In das Echogramm von einer Tiefenstreuschicht sind die Aufzeichnungen eines fernmeldenden Tiefenschreibers eingetragen, um zu zeigen, in welchem Horizont die Planktonröhre gefischt hat. (Nach Kinzer, unveröffentlicht)

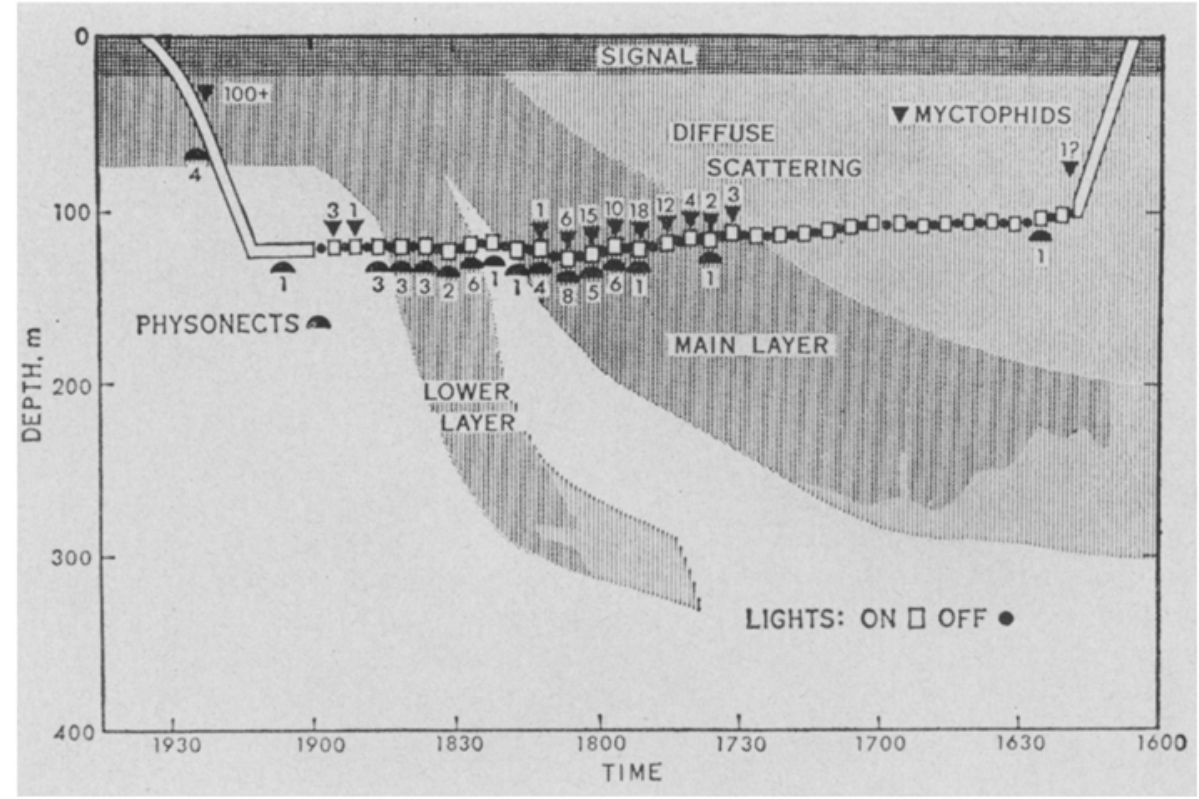

Abb. 8: In das Echogramm, das vom Begleitschiff aufgenommen worden ist, wurde die Tauchtiefenlinie mit Hell- und Dunkelzeiten eingetragen. Die Anzahl der während der Hellzeit beobachteten Myctophiden ist oberhalb (Dreiecke), die der Siphonophoren unterhalb (Halbkreise) der Linie vermerkt. (Nach BARHAM 1966)

reflektierenden Organismen am Beobachter vorbeiwandern mußten. Um einen scheuchenden oder auch anlockenden Effekt des Lichtes zu vermeiden, wurden die Scheinwerfer nach jeweils 3 Minuten Dunkelheit für 2 Minuten eingeschaltet. Die Anzahl der während der Hellzeit beobachteten Tiere ist in die Abbildung 8 eingetragen, und zwar für die Myctophiden markiert durch Dreiecke oberhalb der Tauchtiefenlinie und für 
die physonectiden Siphonophoren durch Halbkreise unterhalb derselben. Das Auftreten der den Schall reflektierenden Tiere konzentrierte sich demnach deutlich auf die Zeit, in der die Reflektionsschicht auf dem Echographen die Tiefe des Tauchbootes passierte. Vorher, beim Abstieg des Bootes und etwa bis $17^{30} \mathrm{Uhr}$, wurden keine Tiere beobachtet. In der Hauptechoschicht fanden sich zahlreiche Vertreter beider Tiergruppen,

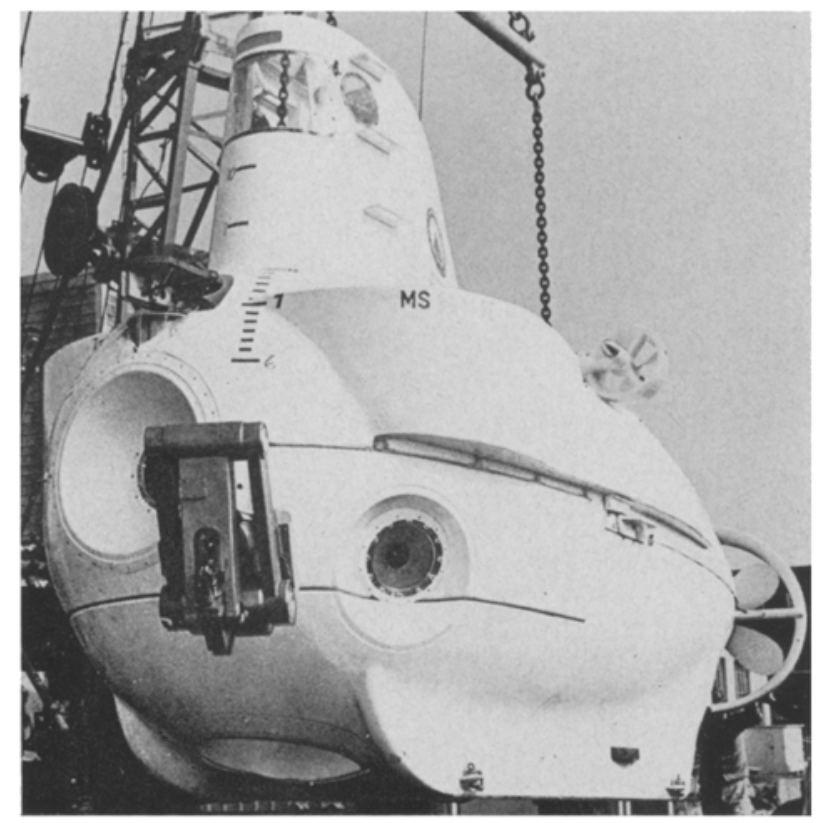

Abb. 9: Alvin, das Tauchboot yom Woods Hole Oceanographic Institute, Office of Naval Research. Am Bug befindet sich die Greifvorrichtung. (Aus Undersea Vehicles for Oceanography)

während die zweite Echoschicht offenbar nur von Siphonophoren gebildet wurde. Nachdem auch diese Schicht am Tauchboot vorbeigewandert war, traten nur noch einzelne Tiere auf. Beim Auftauchen des Bootes - allerdings unter Dauerlicht - wurden dicht unter der Oberfläche zahlreiche Myctophiden gesehen.

Außer diesen beiden Tiergruppen, die eindeutig in enger Beziehung zur Tiefenstreuschicht standen, wurden Tintenfische, Heteropoden, Euphausiaceen, pelagische Decapoden und nicht näher bestimmte Fische beobachtet, die auf Grund ihrer Größe als Echogeber angesehen werden konnten, jedoch mit der aufsteigenden Schicht nicht in Zusammenhang zu bringen waren.

Unsere Darstellung über Untersuchungen von Plankton und Echostreuschichten mit Hilfe von Tauchgeräten sollte zeigen, daß das Tauchen als Methode nicht nur bei biologischen Benthosarbeiten (z. B. Vaissiere \& CARPIne 1964) und in der Meeresgeologie (z. B. Giermann 1966) zu verwenden ist. Eine große Anzahl weiterer Fragestellungen aus dem pelagischen Bereich ließe sich durch das Tauchen lösen: (1) Verhaltensweisen von Fischen bei der Nahrungssuche und -aufnahme, beim Laichen, auf Wanderungen, vor dem Netz, Schwarmbildung und -verhalten. (2) Schwarmbildung 


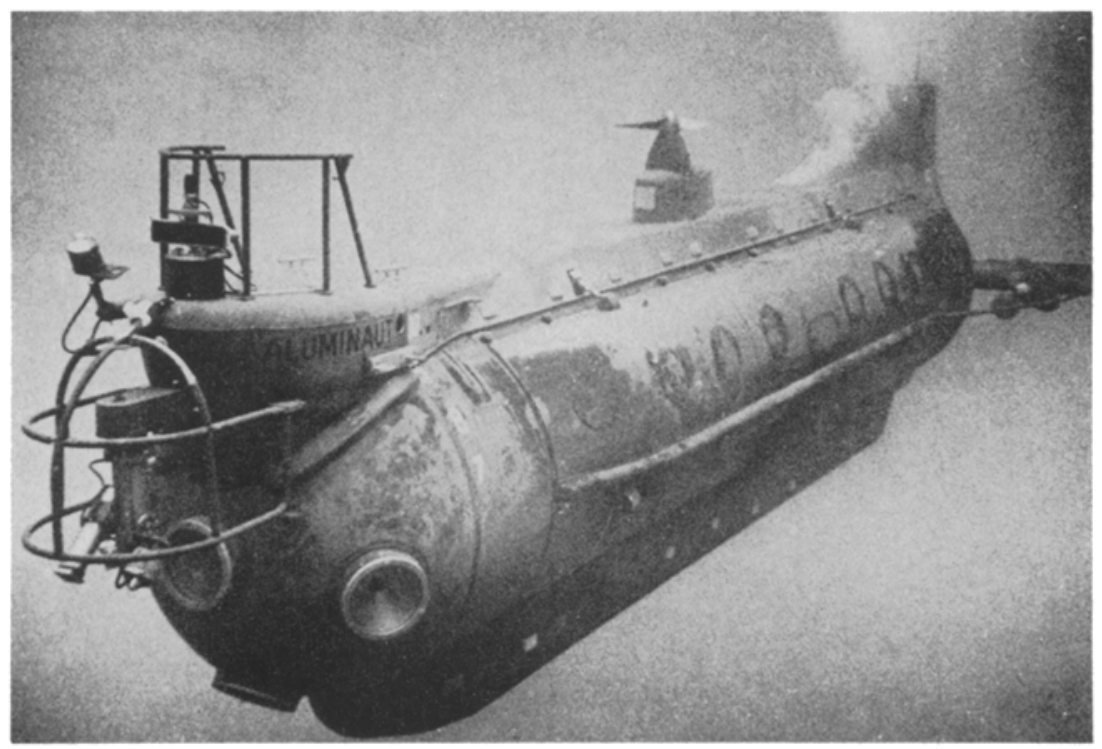

Abb. 10: Aluminaut von Reynolds International Inc., Reynolds submarine services Corp. (Aus Undersea Vebicles for Oceanography)

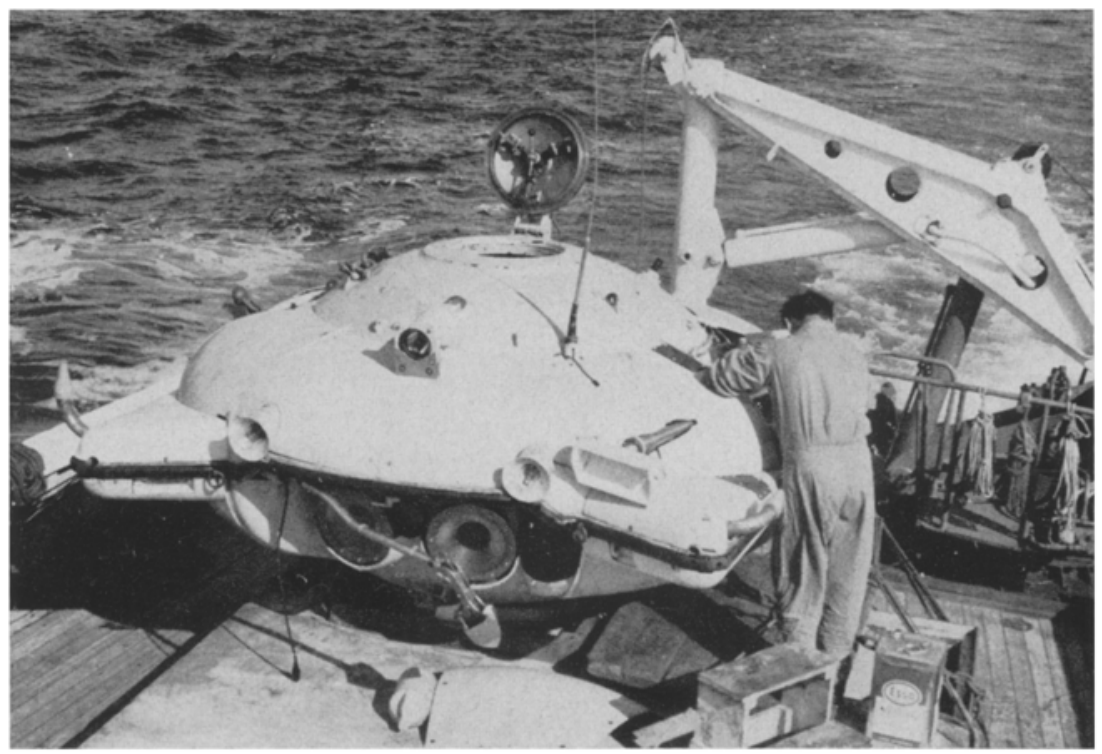

Abb. 11: Cousteau's Soucoupe Plongeante an Bord der Calypso. (Original von Thiel)

bei Fischbrut und Plankton, Verhalten des Schwarmes und einzelner Individuen. (3) Vertikalwanderungen und -verbreitung von Plankton und Fischen. (4) Beobachtung von Tiefsee-Tintenfischen. 
Außer den bereits erwähnten Tauchbooten Bathyscaph und Soucoupe Plongeante wird es sehr bald eine beachtliche Anzahl von Tauchkörpern geben, die für wissenschaftliche Untersuchungen eingesetzt werden können. In der Sowjetunion wird seit längerer Zeit das umgebaute U-Boot "Severyanka“ für fischereiliche Untersuchungen verwendet (z. B. Ryzhenko 1961, u. a.), und „Atlanta-1“ ist ein neues Tauchboot, das

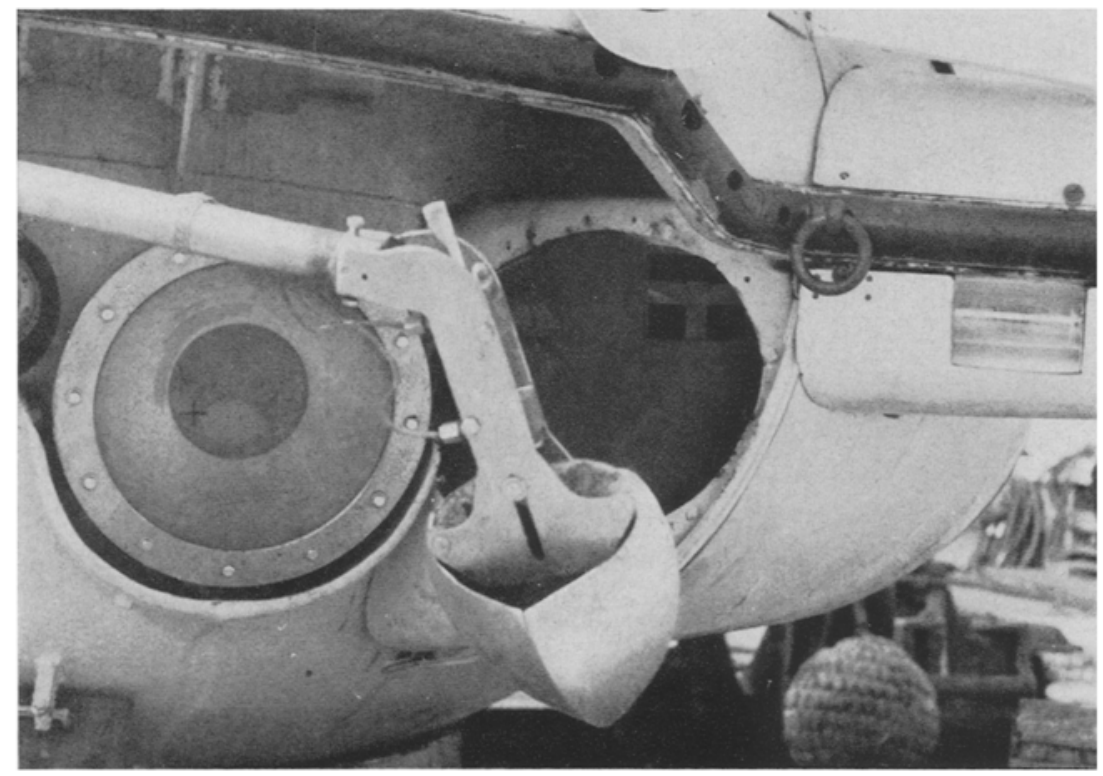

Abb. 12: Die Greifzange der Soucoupe Plongeante. Rechts dahinter befindet sich die Ơffnung des Sammelraumes, die durch eine herabhängende Gummiplatte (schwarz) verschlossen ist. (Original von THIEL)

bis zu Tiefen von $200 \mathrm{~m}$ tauchen kann (Undersea Vehicles 1965a). Die Hauptentwicklung findet jedoch in Amerika statt, wo zur Zeit über 20 Tauchboote von verschiedenen Firmen geplant, gebaut und erprobt werden. Von einigen sind die technischen Daten in der Broschüre "Undersea Vehicles for Oceanography“ (Undersea Vebicles 1965b) zusammengestellt. Von den heute bereits benutzten Tauchbooten erreicht "Alvin“ vom Woods Hole Oceanographics Institute (Abb. 9) die größte Tiefe mit $2000 \mathrm{~m}$, und von den Booten, die sich in der Erprobung befinden, wird "Aluminaut" von Reynolds International Ltd. bis $5000 \mathrm{~m}$ Tiefe tauchen können (Abb. 10).

Für die Bearbeitung biologischer Probleme genügt die Beobachtung allein nicht, es muß auch Gelegenheit gegeben sein, beliebige Proben entnehmen zu können. Das ist indessen bisher nicht der Fall. Alle bislang eingesetzten Tauchboote sind zur Probennahme nur mit einer völlig unzureichenden Greifzange ausgerüstet, wie sie in den Abbildungen 11 und 12 von Cousteaus Soucoupe Plongeante wiedergegeben ist. Hierzu gehört ein ebenfalls unzulänglicher Sammelraum hinter einer herabhängenden Gummiplatte, in dem die Proben während des Tauchganges aufbewahrt werden (Abb. 12). 


\section{GERATEFORDERUNGEN FUR DEN EINSATZ VON TAUCHBOOTEN BEI BIOLOGISCHEN UNTERSUCHUNGEN}

Im folgenden sind die wichtigsten Vorschläge für die biologische Geräteausrüstung von Tauchbooten zusammengestellt. Neben den Planktongeräten werden auch solche für die Entnahme von Bodenproben erwähnt, da für deren Entnahme ebenfalls keine Geräte vorhanden sind. (1) Für Planktonuntersuchungen müssen Möglichkeiten geschaffen werden, mit Planktonnetzen verschiedener Typen und mit Planktonpumpen qualitative und quantitative Proben nehmen und mit einem Gerät entsprechend dem Planktonrecorder (LONGHURst et al. 1966) die Vertikalverteilung der Organismen feststellen zu können. (2) Zur Aufnahme des Benthos, ebenfalls qualitativ und quantitativ, müssen Dredgen, Bodengreifer und Bodensauger (REYs et al. 1966; von Massé 1967 nochmals beschrieben) vorhanden sein. (3) Sowohl für die Probennahme vom Plankton als auch vom Benthos muß dafür gesorgt werden, daß diese Geräte nacheinander und mehrfach bei einem Tauchgang eingesetzt und die Proben in getrennten Behältern verwahrt werden können. Für Planktonproben kommen dafür in Frage: (a) "multiple plankton sampler" nach Bé (1962), (b) das Multinetz von Simonsen (1967) für Vertikalfänge, das am Einleiterkabel gefahren wird und (c) die Wechselvorrichtung von KINZER (in Erprobung, unveröffentlicht), bei der 6 an einem Revolver sitzende Fangbecher über einen Fallgewichtskontakt oder elektrische Auslösung durch einen Motor bewegt werden. Die Bodenproben müssen in einem zu entwickelnden Magazin untergebracht werden können, damit sie isoliert voneinander und ungestört die Meeresoberfläche erreichen. (4) Für Untersuchungen über den Energiehaushalt unter verschiedenen Temperatur- und Druckbedingungen werden lebende Tiefseetiere benötigt. Um diese lebend an die Meeresoberfläche bringen zu können, sind Sammelgeräte mit temperatur- und druckgeschützten Kammern erforderlich.

Die Entwicklung der empfohlenen Einrichtungen dürfte keine unlösbaren Schwierigkeiten bereiten, da für die Funktionen dieser Geräte in den Tauchbooten genügend Energie vorhanden ist oder untergebracht werden kann.

\section{ZUSAMMENFASSUNG}

1. Das Tauchen als Methode zur Untersuchung von Plankton und Echostreuschichten wird durch vier Beispiele erläutert: (a) Visuelle Beobachtungen an Wasserschichtungen und Grenzschichten durch Schwimmtaucher. (b) Untersuchung von Echostreuschichten durch Freitaucher, wobei sich ergab, daß angesammeltes biogenes Material in den untersuchten Sprung- beziehungsweise Streuschichten die Schallreflektion nicht beeinflußt. (c) Beobachtung von Großplankton und Feststellung von Planktonund Sestonkonzentrationen beim Tauchen mit dem Bathyscaph. (d) Untersuchung der Tiefenstreuschicht (deep scattering layer) durch Beobachtung der Vertikalwanderung bestimmter Arten des Großplanktons mit den Tauchbooten Bathyscaph und Soucoupe Plongeante. Physonectide Siphonophoren und Myctophiden standen in deutlicher Beziehung zur Tiefenstreuschicht und wurden als Echogeber erkannt.

2. Die Möglichkeiten, von Tauchbooten aus quantitative und qualitative Proben von 
Plankton und auch vom Benthos zu nehmen, sind zur Zeit noch unzureichend. Die Entwicklung entsprechender Geräte für den wahlweisen und mehrfachen Einsatz bei demselben Tauchgang wird empfohlen.

\section{ZITIERTE LITERATUR}

BANNER, A. H., 1955. Note on a visible thermocline. Science, N. Y. 121, 402-403.

Barfam, E. G., 1963a. Siphonophores and the deep scattering layer. Science, N. Y. 140, $826-828$.

- 1963b. The deep scattering layer as observed from the Bathyscaph 'Trieste'. Int. Congr. Zool. 16 (Washington, D. C.).

- 1966a. Deep scattering layer, migration and composition: observations from a diving saucer. Science, N.Y. 151, 1399-1403.

- 1966b. Deep scattering layer observations from submersible vehicles. Int. oceanogr. Congr. 2 (Moscow) 18, 19-20 (Abstr.).

BÉ, A. W. H., 1962. Quantitative multiple opening- and closing plankton samplers. Deep Sea Res. 2, 144-151.

Bernard, F., 1958. Plancton et benthos observés durant trois plongées en bathyscaphe au large de Toulon. Annls Inst. oceanogr., Monaco 35, 287-326.

- 1962. Contribution du bathyscaphe à l'étude du plancton: avantages et inconvénients. In: Contributions to Symposium on zooplankton production. Ed. by J. H. Fraser \& J. Corlett. No 4. Host \& Fils, Copenhague (Rapp. P.-v. Réun. Cons. perm. int. Explor. Mer 153, 25-28).

Dietz, R. S., 1959. 1100-m dive in the bathyscaphe 'Trieste'. Deep Sea Res. 4, 94-101.

Giermann, G., 1966, Tauchkugel 'Soucoupe plongeante' und Fotoschlitten 'Troika', zwei neue Werkzeuge für die geologische Unterwasserkartierung. Dt. bydrogr. Z. 19, (4): 170-177.

KINZER, J., 1968. Organismen aus Echostreuschichten in extrem sauerstoffarmem Wasser. In: 'Meteor' Forschungsergebnisse. Hrsg. von der Deutschen Forschungsgemeinschaft. Reihe D: Biologie. Borntraeger, Berlin-Nikolassee (in Vorbereitung).

LENZ, J., 1965. Zur Ursache der an die Sprungschicht gebundenen Echostreuschicht in der westlichen Ostsee. Ber. dt. wiss. Kommn Meeresforsch. 18, 111-161.

Limbaugh, C. \& Rechnitzer, A. B., 1955. Visual detection of temperature-density discontinuities in water by diving. Science, N.Y. 121, 395-396.

Longhurst, A. R., Reith, A. D., Bower, R. E. \& Seibert, D. L. R., 1966. A new system for the collection of multiple serial plankton samples. Deep Sea Res. 13, 213-222.

Massè, H., 1967. Intérêt de l'emploi de la «suceuse hydraulique» de C. E. Brett pour les prélèvements quantitatifs dans les substrats meubles infralittoraux, en plongée. Helgoländer wiss. Meeresunters. 15, 500-505.

PÉrès, J.-M., 1958a. Trois plongées dans le canyon du Cap Sicié, effectués avec le bathyscaphe F.N.R.S. III de la Marine Nationale. Bull. Inst. oceanogr. Monaco 55 (1115), 1-21.

- 1958b. Remarques générales sur un ensemble du quinze plongées effectuées avec le bathyscaphe F. N. R. S. III. Annls Inst. oceanogr., Monaco 35, 259-286.

Piccard, J. \& Dietz, R. S., 1957. Oceanographic observations by the bathyscaphe 'Trieste' (1953-1956). Deep Sea Res. 4, 221-229.

ReYs, J. P. \& True, R., 1966. Un nouvel appareil de prélèvement quantitatifs des substrats meubles. Int. oceanogr. Congr. 2 (Moscow) 350, 298-299 (Abstr.).

Ryzhenko, M. I., Sokolov, O. A., Zolotov, S. V. \& Khromov, N. S., 1961. Sixth scientificresearch voyage of the submarine 'Severyanka'. (Transl. from the Russian.) Deep Sea Res. 10, 775-778, 1963. (Aus: Okeanologiya 1 (6), 1094-1096.)

SIMONSEN, R,, 1967. „Multinetz", ein Mehrfachschließnetz für Phytoplankton. In: 'Meteor' Forschungsergebnisse. Hrsg. von der Deutschen Forschungsgemeinschaft. Reihe D: Biologie. Borntraeger, Berlin-Nikolassee, No 1 (im Druck). 
TRÉGOUbofF, G., 1958. La bathyscaphe au service de la planctonologie. Annls Inst. oceanogr., Monaco 35, 327-341.

- 1962. Prospection biologique sous-marine dans la region de Villefranche-sur-Mer en janvier 1961. Bull. Inst. oceanogr., Monaco 59 (1226), 1-14.

Undersea vehicles, 1965a. Undersea vehicle 'Atlanta 1'. Under Sea Technol. 6 (2), 29.

- - 1965b. Undersea vehicles for oceanography. Publ. by the Interagency Committee on Oceanography of the Federal Council for Science and Technology, Washington, $81 \mathrm{pp}$. (Pamphlet 18.)

Varssiere, R. \& Carprne, C., 1964. Contribution à l'étude bionomique de la Méditerranée occidentale (Côte du Var et des Alpes maritimes; Côte occidentale de Corse). Fasc. 4: Compte rendu de plongées en soucoupe plongeante SP 300 (région A1). Bull. Inst. oceanogr. Monaco 64 (1314), 1-14.

\section{Diskussion im Anscbluß an den Vortrag L.enz \& ThIEL}

ANGEL: Several workers have shown that the position of deep scattering layers is a function of the frequency of the sound used. Currie, for example, in a recent Zoological Society Symposium (in press) has described how layers were observed at all depths off Madeira depending on the frequency used. He also describes experiments with lights showing how the layer disappears immediately when the light intensity is increased.

Thiel: Barham hatte bereits früher nachgewiesen, daß er mit der bei diesen Untersuchungen benutzten Frequenz ein Echo von den Siphonophoren und den Myctophiden erhielt. Außerdem beobachtete er eine Anzahl anderer Tierarten, die auf Grund ihrer Größe wohl als Echogeber in Frage gekommen wären, aber nicht in eine Beziehung zur aufgezeichneten Echostreuschicht gebracht werden konnten.

Michanek: Daß Sie hier etwa dasselbe Plankton über und unter der Sprungschicht gefunden haben, kann ja auch zufällig gewesen sein. Es handelt sich doch um sehr verschiedene Wassermassen. An der schwedischen Westküste habe ich beispielsweise mehrmals beobachtet, wie Cyanea im oberen warmen Wasser des baltischen Stroms ziemlich häufig ist, während gleich unter der Sprungschicht große Mengen an Aurelia vorhanden sind, und zwar in solcher Individuendichte, daß es wie ein Boden aussieht oder wie eine zweite Fläche.

THIEL: Ihre Feststellungen entsprechen unseren Beobachtungen an Halitholus cirratus und an Bolinopsis infundibulum. Die verschiedenen Möglichkeiten einer Trübung der Schichten hatten wir schon erwähnt. Wir haben hier bewußt ein Beispiel vorgetragen, in dem die Echostreuschicht eindeutig durch den Dichtesprung, also rein physikalisch, erklärbar ist. Beim gleichzeitigen Auftreten von Plankton- oder Sestonansammlungen in der Streuschicht hätte man nicht sicher darauf schließen dürfen, daß diese Streuschicht ein rein physikalisches Phänomen wäre.

MichaneK: Ja, aber das Gegenteil kommt auch vor, etwa im Hafenwasser, wo sich große Mengen toter Planktonten an der Sprungschicht ansammeln können. Ein weiteres Absinken ist dann nicht möglich.

THIEL: Sicher, Plankton- und Sestonkonzentrationen an der Sprungschicht können vorkommen, jedoch scheint die Schallstreuung nicht dadurch beeinflußt zu werden. Das Echo wird offenbar nur vom temperatur- und salzgehaltsabhängigen Dichtesprung erzeugt.

GREVE: Haben Sie bei Beobachtungen an Einzelplanktern in bewegtem Wasser oberhalb der Sprungschicht eine Reaktion von Tieren auf die in diesem Bereich vorkommenden Vertikalströme beobachtet, die eine gezielte Ausnutzung der Strömung sein könnten? Ich glaube, ähnliches vor Helgoland beobachtet zu haben und möchte wissen, ob Sie eine solche Möglichkeit bei Ihren weitergehenden Beobachtungen berücksichtigt haben?

THIEL: Strömungen, wie Sie sie erwähnen, werden im Helgoländer Bereich vermutlich Auftriebsströmungen sein, die durch den Tidenstrom an Bodenerhebungen entstehen. Die Strömun- 
gen in der westlichen Ostsee sind dagegen so gering, daß keine entsprechenden Beobachtungen gemacht werden konnten.

Hempel: Wie kann man befriedigende Zählungen zur Bestimmung der Planktondichte vom absinkenden Bathyscaph durchführen? Die Angabe von BERNARD über 200 Organismen/Sekunde hat in diesem Zusammenhang erhebliches Aufsehen erregt.

THIEL: Die Geschwindigkeit des absinkenden Bathyscaphs wird möglichst gering und gleichmäßig gehalten. Da von den Scheinwerfern nur ein bestimmter Kegel ausgeleuchtet wird, braucht jeweils nur ein kleines Wasservolumen mit seinem Planktoninhalt beobachtet zu werden. Mit einiger Erfahrung können doch wohl brauchbare Werte erzielt werden.

Bougrs: A propos de la question de Dr. Hempel concernant les numérations de F. Bernard au cours de la descente du Bathyscaphe je peux préciser que celui-ci utilisait un instrument limitant le champ observé et multipliait ensuite par un facteur correspondant au rapport du champ observé au champ total de visée.

LindQuist: Kann der von den Autoren konstruierte Apparat zum Studium der Mikrostratifikation der Dichtesprungschicht wirklich repräsentative Proben des Planktons entnehmen? Werden beispielsweise Copepoden in genügender Menge gesammelt?

Lenz: Die mit diesem Apparat genommenen Proben sind nur für den Phytoplankton- und Sestongehalt repräsentativ, da das Flaschenvolumen nur $300 \mathrm{ml}$ beträgt. Die Häufigkeit der Copepoden wurde mit anderen Geräten untersucht, mit einer Planktonpumpe und mit dem "Hai" (high speed sampler). Es zeigte sich dabei kein Zusammenhang zwischen Zooplanktonkonzentrationen und solchen Echostreuschichtanzeigen, wie wir sie beschrieben haben. 